\title{
PRODUCT SOLUTIONS FOR SIMPLE GAMES. III
}

\section{T. PARTHASARTHY}

1. Introduction. For simple games solution theory, the main object of investigation now is to determine wider classes of solutions for wider classes of games and to develop the structural relationships between solutions of structurally related games. In this paper we obtain partial families of solutions to certain compound games.

2. Definitions and notations. We shall denote a simple game by the symbol $\Gamma(P, W)$ where $P$ is a finite set (players) and $W$ is a collection of subsets of $P$ (the winning coalitions). We demand that $P \in W$ and the empty set is not an element of $W$.

Let $\Gamma\left(P_{1}, W_{1}\right)$ and $\Gamma\left(P_{2}, W_{2}\right)$ be two simple games with $P_{1} \cap P_{2}=\varnothing$ and let $P=P_{1} \cup P_{2}$. Then the product $\Gamma\left(P_{1}, W_{1}\right) \otimes \Gamma\left(P_{2}, W_{2}\right)$ [for simplicity we will write $\left.P_{1} \otimes P_{2}\right]$ is defined as the game $\Gamma(P, W)$ where $W$ consists of all $S \subseteq P$ such that $S \cap P_{i} \in W_{i}$ for $i=1,2$. We will use the same notation $P$ for the number of elements in $P$. By an imputation we mean a nonnegative vector $x$ such that $\sum_{i=1}^{P} x_{i}=1 . A_{P}$ will stand for the collection of all imputations. We recall that a solution of the game $\Gamma(P, W)$ is a set $X$ of imputations such that $X=A_{P}-\operatorname{dom} X$ where $\operatorname{dom} X$ denotes the set of all $y \in A_{P}$ such that for some $x \in X$, the set $\left[i: x_{i}>y_{i}\right]$ is an element of $W$. The notations dom $_{1}$ and dom 2 will be used for domination with respect to special classes $W_{1}$ and $W_{\mathbf{2}}$.

Definition. A parametrized family of sets of imputations

$$
[Y(\alpha): 0 \leqq \alpha \leqq 1]
$$

will be called semimonotone if for every $\alpha, \beta, x$ such that $0 \leqq \alpha \leqq \beta \leqq 1$ and $x \in Y(\beta)$ there exists $y \in Y(\alpha)$ with $\alpha y \leqq \beta x$. [Here $\alpha, \beta$ are scalars, $y, x$ are vectors and $\alpha y \leqq \beta x$ means each one of the coordinates in $\alpha y$ is less than or equal to the corresponding coordinates in $\beta x$.]

Definition. A semimonotonic family is called $\delta$-monotonic $(0 \leqq \delta \leqq 1)$ if for every $\alpha, \beta, y$ such that $\delta \leqq \alpha \leqq \beta \leqq 1$ and $y \in Y(\alpha)$ there exists $x \in Y(\beta)$ with $\alpha y \leqq \beta x$.

We call a 0 -monotonic family fully-monotonic. In general $\delta$ will stand for any positive number with $0<\delta<1$. Let $P=P_{1} \cup P_{2}$ and let

$$
A_{P_{i}}=\left\{x: x \in A_{P}, \sum_{j \in P_{i}} x_{j}=1\right\} \quad \text { for } i=1,2 .
$$

Received by the editors February 10, 1969 and, in revised form, March 24, 1969. 
Definition. Let $Y$ be a solution to the product of simple games $P_{1} \otimes P_{2}$. Call $Y$ a product solution if the following conditions are met.

(i) There exists a semimonotonic family $\left\{Y_{i}(\alpha): 0 \leqq \alpha \leqq 1\right\}$ such that $Y_{i}(\alpha)$ are solutions to $P_{i}$ for all $\alpha$ except $\alpha=1$ where $i=1,2$ and

(ii) $Y=\bigcup_{0 \leqq \alpha \leqq 1} \quad Y_{1}(\alpha) \times_{\alpha} Y_{2}(1-\alpha) \cup Y_{1}^{*} \cup Y_{2}^{*}$ where $Y_{i}^{*}=A_{P_{i}}$ $-Y_{i}(1)-\operatorname{dom}_{i} Y_{i}(1)$ and $Y_{1}(\alpha) \times_{\alpha} Y_{2}(1-\alpha)$ defined as the set $=\left\{z: z=\alpha x_{1}+(1-\alpha) x_{2}\right.$ for some $x_{1} \in Y_{1}(\alpha)$ and $\left.x_{2} \in Y_{2}(1-\alpha)\right\}$.

Definition. Let $\Gamma(P, W)$ be a simple game. Let $X$ be any subset of $A_{P}$. Call $X$ an internally stable set if $X \cap \operatorname{dom} X=\varnothing$. Call $X$ an externally stable set if $X \cup \operatorname{dom} X=A_{P}$. Call $X$ a solution if $X$ is both internally stable and externally stable.

Definition. Let $\Gamma(P, W)$ be a simple game. Let $x, y \in A_{P}$. Let $S \in W$. We say $x$ dominates $y$ via $S$ (in notation $x>y$ via $S$ or $x>_{S} y$ ) if $x_{i}>y_{i}$ for all $i \in S$.

3. A theorem on the simple game $J$. The four-person game $J$ is defined by

$$
J=\Gamma((1,2,3,4),\{124,134,234,1234\})
$$

where members in the curly brackets denote the winning coalitions of the game $J$.

$$
J=M_{123} \otimes B_{4} .
$$

$M_{123}$ denotes the 3-person simple majority game and $B_{4}$ the 1-person pure bargaining game.

In [2] we have proved the following theorem.

Theorem 1. Let $0 \leqq \delta<1$ and let $\left[Y_{1}(\alpha): 0 \leqq \alpha \leqq 1\right]$ be any $\delta$-monotonic family of product solutions except that $Y_{1}(1)$ need not be externally stable to the game $J=M_{123} \otimes B_{4}$. Then

$$
Y=\bigcup_{0 \leqq \alpha \leqq 1} Y_{1}(\alpha) \underset{\alpha}{\times} Y_{2}(1-\alpha) \cup Y_{1}^{*}
$$

is a solution for $J \otimes K$ where $K$ is any arbitrary simple game and $Y_{1}^{*}$ $=A_{4}-Y_{1}(1)-\operatorname{dom}_{1} Y_{1}(1)$ and $Y_{2}(1-\alpha) \equiv Y_{2}$ being any solution to $K$.

REMARK 1. Theorem 1 has its own limitations, as every solution to $J$ need not be a product solution. For example, consider the following solution.

Let $s=(0,1 / 4,1 / 4,1 / 2)$ and let $L=L_{1} \cup L_{2} \cup L_{3}$ where

$$
\begin{aligned}
& L_{1}=\underset{0 \leqq t \leqq 1 / 2}{U}[(1 / 4, x, y, t): x, y \geqq 0 \text { and } x+y=3 / 4-t] ; \\
& L_{2}=\bigcup_{1 / 2<t \leqq 3 / 4}^{\bigcup}[(3 / 4-t, x, y, t): x, y \geqq 0 \text { and } x+y=1 / 4] ;
\end{aligned}
$$




$$
L_{3}=\bigcup_{3 / 4<t \leqq 1}[(0, x, y, t): x, y \geqq 0 \quad \text { and } \quad x+y=1-t] .
$$

Define $H=L \cap$ dom $s$.

Then it can be shown that $\{s\} \cup(L-H)$ is a solution for $J$. For proof of this assertion see Shapley [4]. Furthermore, this is not a product solution to $J$. In this section we are going to show that Theorem 1 remains true if one includes solutions of the type constructed by Shapley [4] containing an arbitrary closed component for the game $J$.

Let $C$ be an arbitrary closed subset of $[0,1)$ and let $S$ be the image of $C$ under the one-one map

$$
u_{4} \in C \rightarrow\left\langle 0, \frac{1-u_{4}}{2}, \frac{1-u_{4}}{2}, u_{4}\right\rangle .
$$

Consider the set $L$ which consists of all imputations of the form

$$
\left\{\left\langle\frac{1-u_{4}-\rho\left(u_{4}, C^{0}\right)}{2}, x, y, u_{4} \backslash: u_{4} \in[0,1]\right\}\right.
$$

where $C^{0}=C \cup\{1\}, \rho\left(u_{4}, C^{0}\right)=\operatorname{Inf}_{u \in C^{0}}\left|u_{4}-u\right|, x, y \geqq 0$ and $x+y$ $=\left[1-u_{4}+\rho\left(u_{4}, C^{0}\right)\right] / 2$.

Let $H=L \cap \operatorname{dom} S$.

The $S \cup(L-H)$ is a solution for $J$. For a proof refer [4]. We will call such solutions $C$-solutions.

Theorem 2. Let $0 \leqq \delta<1$ and let $Y_{1}(\alpha)$ be C-solutions to $J$ (except that $Y_{1}(1)$ need not be externally stable) and $\left[Y_{1}(\alpha): 0 \leqq \alpha \leqq 1\right]$ be $\delta$-monotonic. Then

$$
Y=\bigcup_{0 \leqq \alpha \leqq 1} Y_{1}(\alpha) \underset{\alpha}{\times} Y_{2}(1-\alpha) \cup r_{1}^{*}
$$

is a solution to $J \otimes K$ where $Y_{2}(1-\alpha) \equiv Y_{2}$ is any solution to $K$ and $Y_{1}^{*}=A_{4}-Y_{1}(1)-\operatorname{dom}_{1} Y_{1}(1)$.

REMARK 2. Since $\left\{Y_{1}(\alpha)\right\}$ is semimonotonic it follows that $Y_{1}(1)$ is internally stable and hence $Y_{1}(1) \cup Y_{1}^{*}$ is externally stable. Hence external stability of Theorem 2 can be proved as in the case of Theorem 5 of Shapley (see [3, pp. 282-283]) or as in [1].

REMARK 3. As the family $\left\{Y_{1}(\alpha): 0<\alpha<1\right\}$ with $\bar{Y}_{1}(1)$ [closure of $\left.Y_{1}(1)\right]$ is also semimonotonic, we will assume $Y_{1}(1)$ itself is closed.

Proof of Theorem 2. We will only indicate the proof of internal stability. Suppose there exist $x, y \in X$ such that $x>y$ via $S \in W$. Let $S_{1}=S \cap P_{1}, S_{2}=S \cap P_{2}$ and $x=\alpha x_{1}+(1-\alpha) x_{2}, y=\beta y_{1}+(1-\beta) y_{2}$ where $x_{1}, y_{1} \in A_{4}$ and $x_{2}, y_{2} \in A_{K}$. Since $x>y$ on $S_{1} \cup S_{2}=S$ it follows that $0<\alpha<1$. 
Case 1a. $1>\beta \geqq \alpha$. Since $\alpha>0, \beta>0$. Also $\alpha x_{1}>\beta y_{1}$ on $S_{1}$. Since $Y_{1}(\alpha)$ is semimonotonic there exists $x_{1}^{\prime} \in Y_{1}(\alpha)$ such that $\beta y_{1} \geqq \alpha x_{1}^{\prime}$. Hence $\alpha x_{1}>\beta y_{1} \geqq \alpha x_{1}^{\prime}$, that is $x_{1}>x_{1}^{\prime}$ on $S_{1}$ contradicting the internal stability of $Y_{1}(\alpha)$.

Case $1 \mathrm{~b} . \alpha \geqq \beta>0$. This means $1>1-\beta \geqq 1-\alpha$. Since $\alpha \neq 1$ this case is similar to the previous case except that we have to utilize the semimonotonic property of $Y_{2}(\alpha)$.

Case 2a. $\beta_{1}=1$, that is, $\alpha x_{1}>y_{1}$ on $S_{1}$. If $y_{1} \in Y_{1}(1)$ then using the semimonotonic property of $\left\{Y_{1}(\alpha): 0 \leqq \alpha \leqq 1\right\}$ and $Y_{1}(1)$ [see Remark 3 ], we will arrive at a contradiction. Let if possible $\alpha x_{1}>y_{1}$ on $S_{1}$ with $y_{1} \in Y_{1}^{*}$

$$
S_{1}=\{1,2,4\}, \quad x_{1}=\left(a_{1}, a_{2}, a_{3}, a_{4}\right), \quad y_{1}=\left(\epsilon_{1}, \epsilon_{2}, \epsilon_{3}, \epsilon_{4}\right) .
$$

[For simplicity we are not writing $x_{1}=\left(a_{1} a_{2} a_{3} a_{4} 0000\right)$ etc.] Using the $\delta$-monotonic property and the fact that $Y_{1}(\alpha)$ except $\alpha=1$ are $C$-solutions it can be shown that the following two vectors belong to $Y_{1}(1)$ :

$$
\begin{aligned}
& w_{1}=\left(\frac{1-u_{4}-\rho^{0}}{2}, \frac{1-u_{4}+\rho^{0}}{2}, 0, u_{4}\right) \in Y_{1}(1), \\
& w_{2}=\left(\frac{1-u_{4}-\rho^{0}}{2}, 0, \frac{1-u_{4}+\rho^{0}}{2}, u_{4}\right) \in Y_{1}(1),
\end{aligned}
$$

where $\alpha a_{4}>u_{4}>\epsilon_{4}$ and $\rho^{0} \geqq 0$. If

$$
\left(1-u_{4}-\rho^{0}\right) / 2 \leqq \epsilon_{1}
$$

then $\alpha x_{1}>w_{2}$ via $S_{1}$. This means there exists a vector $x^{\prime}$ such that

$$
\alpha x_{1}>w_{2} \geqq \alpha x^{\prime} \text { via } S_{1} \text { which is impossible. }
$$

We will assume $\left(1-u_{4}-\rho^{0}\right) / 2>\epsilon_{1}$ and hence it follows that $\left(1-u_{4}+\rho^{0}\right) / 2 \leqq \epsilon_{2}$. Otherwise, there will be a contradiction to the fact that $y_{1} \in Y_{1}{ }^{*}$. If $a_{3}>0$, then $\alpha x_{1}>w_{1}$ via 234 which will in turn contradict the internal stability of $Y_{1}(\alpha)$. So we will assume $a_{3}=0$.

Let $C_{\alpha}$ be the closed subset of $[0,1]$ corresponding to $Y_{1}(\alpha)$. If $a_{4} \in C_{\alpha}$ then

$$
\left(0, \frac{1-a_{4}}{2}, \frac{1-a_{4}}{2}, a_{4}\right) \in X_{1}(\alpha) .
$$

Since $a_{3}=0$ it follows that $a_{1}=a_{2}=\left(1-a_{4}\right) / 2$. Hence $\alpha x>w_{1}$ via 124 which is impossible. Let $a_{4} \notin C_{\alpha}$. This means

$$
a_{1}=1-a_{4}-\rho\left(a_{4}, C_{\alpha}^{0}\right) / 2 \text { and } a_{2}=1-a_{4}+\rho\left(a_{4}, C_{\alpha}^{0}\right) / 2
$$

where $C_{\alpha}^{0} \cup C_{\alpha}=\{1\}, \rho\left(a_{4}, C_{\alpha}^{0}\right)>0$ for $a_{4} \notin C_{\alpha}^{0}$ and $C_{\alpha}^{0}$ is compact. Let 
$0<\epsilon<\rho\left(a_{4}, C_{\alpha}^{0}\right)$, then

$$
\left\langle\frac{1-a_{4}-\rho\left(a_{4}, C_{\alpha}^{0}\right)}{2}, \frac{1-a_{4}+\rho\left(a_{4}, C_{\alpha}^{0}\right)-\epsilon}{2}, \epsilon / 2, a_{4}\right\rangle \in Y_{1}(\alpha) .
$$

That is

$$
\alpha\left\{\frac{1-a_{4}-\rho\left(a_{4}, C_{\alpha}^{0}\right)}{2}, \frac{1-a_{4}+\rho\left(a_{4}, C_{\alpha}^{0}\right)-\epsilon}{2}, \epsilon / 2, a_{4}\right\}>w_{1} \text { via } 234
$$

which will once again contradict the internal stability of $Y_{1}(\alpha)$. Consider the case where

$$
a_{3}=\left(1-a_{4}-\rho\left(a_{4}, C_{\alpha}^{0}\right)\right) / 2=0,
$$

that is, $\rho\left(a_{4}, C_{\alpha}^{0}\right)=1-a_{4}$. From the way in which every $C$-solution is constructed it follows that the first two coordinates can run between 0 to $1-a_{4}$. Hence we have

$$
\alpha\left(a_{2}, a_{1}, 0, a_{4}\right)>w_{2} \quad \text { via } 124
$$

which leads to a contradiction. Other cases can be disposed of similarly. Thus the proof is complete.

In fact, one can prove the following theorem, which includes both Theorems 1 and 2.

Theorem 3. Let $0 \leqq \delta<1$ and let $\left\{Y_{1}(\alpha): 0 \leqq \alpha \leqq 1\right\}$ be $\delta$-monotonic solutions to $J$ except that $Y_{1}(1)$ need not be externally stable. Further, suppose each $Y_{1}(\alpha)$, except $\alpha=1$, is either a product solution or a $C$-solution. Then

$$
Y=\bigcup_{0 \leqq \alpha \leqq 1} Y_{1}(\alpha) \underset{\alpha}{\times} Y_{2}(1-\alpha) \cup Y_{1}^{*}
$$

is a solution for $J \otimes K$ where $Y_{2}(1-\alpha) \equiv Y_{2}$ is any solution for $K$ and $Y_{1}^{*}=A_{4}-Y_{1}(1)-\operatorname{dom}_{1} Y_{1}(1)$.

REMARK 4 . We are unable to settle the following question: Suppose $Y_{1}(\alpha)$ is any $\delta$-monotonic family of solutions (nor necessarily product solutions or $C$-solutions) to $J$ except that $Y_{1}(1)$ need not be externally stable. Then will this family yield product solutions to games of the form $J \otimes K$ ? However, one can prove the following partial result in this direction.

THEOREM 4. Let $Y_{1}(\alpha)$ be 1/2-monotonic family of solutions to $J$ 
except that $Y_{1}(1)$ need not be externally stable. Further suppose $\left[Y_{1}(\alpha): \alpha \geqq 1 / 2\right]$ are product solutions. Then

$$
Y=\bigcup_{0 \leqq \alpha \leqq 1} Y_{1}(\alpha) \times Y_{\alpha}(1-\alpha) \cup Y_{1}^{*}
$$

is a solution for $J \otimes K$ where $Y_{2}(1-\alpha) \equiv Y_{2}$ is any solution for $K$.

Proof. We will prove the internal stability of $Y$. For this it is enough if we prove that there does not exist a vector $x \in Y_{1}(\alpha)$ with $\alpha x>y$ via $S$ for some $y \in Y_{1}^{*}$. Let, if possible,

$$
\alpha\left(a_{1}, a_{2}, a_{3}, a_{4}\right)>y=\left(\epsilon_{1}, \epsilon_{2}, \epsilon_{3}, \epsilon_{4}\right) \text { via } 124
$$

where $y \in Y_{1}^{*}$. For every $\beta \in[0,1]$, there exists a set $N_{\beta}$ which belongs to $Y_{1}(1)$. Also $N_{\beta}$ has the following representation.

$$
N_{\beta}=\left[\left\langle\beta\left(C_{\beta}, t, 1-t-C_{\beta}\right), 1-\beta\right\rangle: 0 \leqq t \leqq 1-C_{\beta}\right] .
$$

For details regarding this fact refer [2]. This is a consequence of the fact that $Y_{1}(\alpha)$ is a $1 / 2$-monotonic family and that $\left\{Y_{1}(\alpha): \alpha \geqq 1 / 2\right\}$ are product solutions except $\alpha=1$.

Let us choose a $\beta^{\prime}$ such that $\alpha a_{4}>1-\beta^{\prime}>\epsilon_{4}$ and consider the set $N_{\beta^{\prime}} . \beta^{\prime} C_{\beta^{\prime}}$ cannot be less than or equal to $\epsilon_{1}$ as this will contradict the internal stability of $Y_{1}(\alpha)$. Hence $\beta^{\prime} C_{\beta^{\prime}}>\epsilon_{1}$. Since $y \in Y_{1}^{*}$, it follows, $\beta^{\prime}\left(1-C_{\beta^{\prime}}\right) \leqq \epsilon_{2}, \epsilon_{3}$. So we have the following inequalities

$$
\begin{aligned}
& \alpha a_{4}>1-\beta^{\prime} \\
& \alpha a_{2}>\epsilon_{2} \geqq \beta^{\prime}\left(1-C_{\beta^{\prime}}\right) \geqq \beta^{\prime} / 2 \quad\left[\text { for } C_{\beta^{\prime}} \leqq 1 / 2\right] .
\end{aligned}
$$

Therefore $\alpha>1-\beta^{\prime} / 2 \geqq 1 / 2$. Since $\alpha>1 / 2$ and $Y_{1}(\alpha)$ is $1 / 2$-monotonic, we arrive at a contradiction with regard to the assumption that $y \in Y_{1}^{*}$. Let if possible

$$
\begin{aligned}
N_{\beta^{\prime}}= & \left(\beta^{\prime} / 2, \beta^{\prime} / 2,0,1-\beta^{\prime}\right) \\
& \cup\left(\beta^{\prime} / 2,0, \beta^{\prime} / 2,1-\beta^{\prime}\right) \\
& \cup\left(0, \beta^{\prime} / 2, \beta^{\prime} / 2,1-\beta^{\prime}\right) .
\end{aligned}
$$

As before we have,

$$
\beta^{\prime} / 2>\epsilon_{1}, \quad \beta^{\prime} / 2 \leqq \epsilon_{2}, \quad \alpha x_{4}>1-\beta^{\prime}, \quad \alpha x_{2}>\beta^{\prime} / 2 \quad \text { or } \alpha>1 / 2 .
$$

This once again contradicts the hypothesis that $y \in Y_{1}^{*}$. Similarly other cases can be disposed of and thus the proof is complete.

REMARK 5. We suspect Theorem 4 might be true if we replace $1 / 2$ by any positive $\delta$ near 1 . 
4. A theorem on simple majority game. Let $\Gamma(N, W)$ be an $n$-person simple game. $N=\{1,2, \cdots, n\}$. We are going to assume that the only winning coalitions are those which contain $(n-1)$ elements. That is $S \in W$ means $S=N$ or $S$ contains $(n-1)$-elements from $N$.

Let $Y_{1}(1) \subset A_{N}$ be an internally stable set not necessarily an externally stable set. Assume $Y_{1}(1)$ to be a closed set. Let

$$
Y_{1}^{*}=A_{N}-Y_{1}(1)-\operatorname{dom}_{1} Y_{1}(1) .
$$

Let $y \in E$. Define $y(S)=\sum_{i \in S} y_{i}$ for $S \in W$ where $y=\left(y_{1}, y_{2}, \cdots, y_{N}\right)$. Let $a_{S}=\operatorname{Inf}\left[y(S): y \in Y_{1}^{*}\right]$ for $S \in W$ and $\delta_{0}=\operatorname{Min}\left[a_{S}: a_{S}>0\right]$. If $a_{S} \equiv 0, \forall S \in W$, we take any $\delta \in[0,1)$.

TheOREM 5. Let $\left\{Y_{1}(\alpha): 0 \leqq \alpha \leqq 1\right\}$ be $\delta_{0}$-monotonic (where $\delta_{0}$ is defined as above) family of solutions except that $Y_{1}(1)$ need not be externally stable to $\Gamma(N, W)$. Then

$$
Y=\bigcup_{0 \leqq \alpha \leqq 1} Y_{1}(\alpha) \underset{\alpha}{\times} Y_{2}(1-\alpha) \cup Y_{1}^{*}
$$

is a solution to $N \otimes K$ where $Y_{2}(1-\alpha) \equiv Y_{2}$ any solution to the simple game $K$.

Proof. We need only establish that there does not exist a vector $x \in Y_{1}(\alpha)$ with $\alpha x>y$ via $S$ for some $y \in Y_{1}^{*}$. Let if possible $\alpha x>y$ via $S$. We will consider two cases according as $a_{S}=0$ or $a_{S}>0$.

Case 1. $a_{S}=0$. We will take without loss of generality $S$ $=\{1,2, \cdots, n-1\}$. Let $u=(0,0,0, \cdots, 0,1)$. Since $a_{S}=0$ and $Y_{1}(1) \cup Y_{1}^{*}$ is a closed set, it follows that $u \in Y_{1}(1) \cup Y_{1}^{*}$. Also we have

$$
\alpha x>u \text { via } S \quad \text { (for } \alpha x>y \text { via } S \text { ). }
$$

Now, $u \notin Y_{1}(\beta)$ for any $\beta \geqq \alpha$ otherwise internal stability of $Y_{1}(\alpha)$ will be contradicted. This simply means $u \in Y_{1}^{*}$. Let $\beta>\delta$. There exists a vector $w \in Y_{1}(\beta)$ with $w>u$ via $S$. That is, $\beta w>u$ via $S$. Since the family $Y_{1}(\alpha)$ is $\delta$-monotonic, one can find a vector $v \in Y_{1}(1)$ with

$$
v \geqq \beta w>u \text { via } S, \quad v>u \text { via } S \text {. }
$$

This means $u \notin Y_{1}(1) \cup Y_{1}^{*}$ contradicting the assumption that $u \in Y_{1}(1) \cup Y_{1}^{*}$. Hence we are through in this case.

Case 2. $a_{S}>0$. If $a_{S}=1$, then $\alpha x>y$ via $S$ is impossible. So we assume $0<a_{S}<1$. This means $0<\delta<1$. It is not hard to check that $\alpha>y(S) \geqq a_{S} \geqq \delta$. Since the family is $\delta$-monotonic, we can find a vector $v$ in $Y_{1}(1)$ with $v \geqq \alpha x$. That is $v>y$ via $S$ which once again contradicts the assumption regarding $y$. This completes the proof of Theorem 5 . 
We will close this section with one more theorem on product solutions for simple games.

Theorem 6. Let $\left\{Y_{i}(\alpha): 0 \leqq \alpha \leqq 1\right\}$ for $i=1,2$ be semimonotonic families of solutions to simple games $\Gamma\left(P_{i}, W_{i}\right)$ except that $Y_{i}(1)$ need not be externally stable. Suppose there exist two vectors $u, v$ where $u \in Y_{1}(1), v \in Y_{2}(1)$ with $u \geqq \delta$ and $v \geqq \delta$. [Here each one of the coordinates of $u$ and $v$ are greater than or equal to $\delta$.] Further, suppose the families to be $\delta$-monotonic. Then

$$
Y=\bigcup_{0 \leqq \alpha \leqq 1} Y_{1}(\alpha) \times Y_{\alpha}(1-\alpha) \cup Y_{1}^{*} \cup Y_{2}^{*}
$$

is a solution to $P_{1} \otimes P_{2}$ where $Y_{i}^{*}=A_{P_{i}}-Y_{i}(1)-\operatorname{dom}_{i} Y_{i}(1)$.

REMARK 6. This theorem is a slight variation of Theorem 4, of [1]. The limitation of this theorem is that $\delta$ tends to be small as the number of players in the component games increase.

5. An example of a product solution. The five-person game $F$ is defined by $F=\Gamma(12345,\{1235,1245,1345,2345,12345\})$ where members in the curly brackets denote the winning coalitions of the game $F$.

$$
F=M_{1234} \otimes B_{5}
$$

where $M_{1234}$ denotes the 4 -person simple majority game and $B_{5}$ the 1-person pure bargaining game. Define, for $0 \leqq \alpha \leqq 1 / 3$

$$
Y_{1}(\alpha)=\bigcup_{0 \leq \beta \leq 1} Y(\beta)
$$

where

$$
\begin{aligned}
Y(\beta)= & \left\{\left(\beta^{2} / 3, x, y, z, 1-\beta\right): x, y, z \geqq 0 \text { and } x+y+z=\beta-\beta^{2} / 3\right\} \\
& \bigcup\left\{0, \frac{1}{3}, \frac{1}{3}, \frac{1}{3}, 0\right\} .
\end{aligned}
$$

Define, for $1 / 3<\alpha \leqq \alpha_{0}$ where $\alpha_{0}$ is so chosen that

$$
\begin{gathered}
\alpha_{0}\left(1-4 / 9\left(1+\alpha_{0}\right)\right)=2 / 3, \\
Y_{1}(\alpha)=\bigcup_{0 \leq \beta \leq 1} Y(\beta)
\end{gathered}
$$

where

$$
\begin{array}{r}
Y(\beta)=\left\{\left(\frac{4}{9} \frac{\beta^{2}}{1+\alpha}, x, y, z, 1-\beta\right): x, y, z \geqq 0 \text { and } x+y+z\right. \\
\left.=\beta-\frac{4}{9} \cdot \frac{\beta^{2}}{1+\alpha}\right\} .
\end{array}
$$


For $\alpha_{0}<\alpha<1$ define

$$
Y_{1}(\alpha)=\bigcup_{0 \leq \beta \leq 1} Y(\beta)
$$

where

$$
\begin{aligned}
Y(\beta)=\left\{\left[(1-2 / 3 \alpha) \beta^{2}, x, v, z, 1-\beta\right]: x, y, z \geqq 0\right. & \text { and } x+y+z \\
& \left.=\beta-(1-2 / 3 \alpha) \beta^{2}\right\} .
\end{aligned}
$$

For $\alpha=1$, define

$$
Y_{1}(\alpha)=\bigcup_{0 \leq \beta \leq 1} Y(\beta)
$$

where

$$
Y(\beta)=\left\{\left(\beta^{2} / 3, x, y, z, 1-\beta\right): x, y, z \geqq 0 \text { and } x+y+z=\beta-\beta^{2} / 3\right\} .
$$

Now it is not hard to check that the family $\left[Y_{1}(\alpha): 0 \leqq \alpha \leqq 1\right]$ is semi monotonic and that each $Y_{1}(\alpha)$ is a solution to the game $F$ except that $Y_{1}(1)$ is not externally stable because $\left(0, \frac{1}{3}, \frac{1}{3}, \frac{1}{3}, 0\right)$ is not an element of $Y_{1}(1) \cup$ dom $Y_{1}(1)$. If $Y_{1}^{\prime}(1)=Y_{1}(1) \cup\left\{\left(0, \frac{1}{3}, \frac{1}{3}, \frac{1}{3}, 0\right)\right\}$, then $Y_{1}^{\prime}(1)$ is a solution to $F$. But $Y_{1}(\alpha)$ for $0 \leqq \alpha<1$ together with $Y_{1}^{\prime}(1)$ is not semimonotonic. Further it is not hard to check that the family $\left[Y_{1}(\alpha): 0 \leqq \alpha \leqq 1\right]$ is $2 / 3$-monotonic but not fully monotonic. Moreover, all the conditions of Theorem 5 are satisfied. Hence, this family can be used to produce solutions to games of the form $F \otimes K$.

Acknowledgment. The author is grateful to Dr. L. S. Shapley for his interest in this work, and to the referee for some useful suggestions.

\section{REFERENCES}

1. T. Parthasarathy, A note on compound simple games, Proc. Amer. Math. Soc. 17 (1966), 1334-1340.

2. - Product solutions for simple games. II, Proc. Amer. Math. Soc. 20 (1969), $107-114$.

3. L. S. Shapley, Solutions of compound simple games, Ann. of Math. Studies, no. 52, Princeton Univ. Press, Princeton, N.J., 1964.

4. - - A solution containing an arbitrary closed component, Ann. of Math. Studies, no. 40, Princeton Univ. Press, Princeton, N.J., 1959.

Case Western Reserve University 